\title{
Multi-Fidelity Modeling-Based Structural Reliability Analysis with the Boundary Element Method
}

\author{
Llewellyn Morse*, Zahra Sharif Khodaei and M. H. Aliabadi \\ Department of Aeronautics \\ Imperial College London \\ South Kensington Campus \\ Roderic Hill Building, Exhibition Road \\ $S W 7$ 2AZ, London, UK \\ *llewellyn.morse12@imperial.ac.uk
}

Received 5 June 2017

Accepted 7 August 2017

Published 31 August 2017

\begin{abstract}
In this work, a method for the application of multi-fidelity modeling to the reliability analysis of 2D elastostatic structures using the boundary element method (BEM) is proposed. Reliability analyses were carried out on a rectangular plate with a center circular hole subjected to uniaxial tension using Monte Carlo simulations (MCS), the first-order reliability method (FORM), and the second-order reliability method (SORM). Two BEM models were investigated, a low-fidelity model (LFM) of 20 elements and a high-fidelity model (HFM) of 100 elements. The response of these models at several design points was used to create multifidelity models (MFMs) utilizing second-order polynomial response surfaces and their reliability, alongside that of the LFM and the HFM, was evaluated. Results show that the MFMs that directly called the LFM were significantly superior in terms of accuracy to the LFM, achieving very similar levels of accuracy to the HFM, while also being of similar computational cost to the LFM. These direct MFMs were found to provide good substitutes for the HFM for MCS, FORM, and SORM.
\end{abstract}

Keywords: Multi-fidelity modeling; boundary element method (BEM); reliability analysis; Monte Carlo simulation (MCS); first-order reliability method (FORM); second-order reliability method (SORM).

\section{Introduction}

All engineering parameters have uncertainties in their values, it is therefore important that these are considered during the design process. Failure to consider uncertainties can lead to the design failing or becoming overengineered, requiring large safety factors. As stated by Huang et al., ${ }^{1}$ adequate performance cannot be

*Corresponding author.

This is an Open Access article published by World Scientific Publishing Company. It is distributed under the terms of the Creative Commons Attribution 4.0 (CC-BY) License. Further distribution of this work is permitted, provided the original work is properly cited. 
guaranteed when there is uncertainty. Therefore, a probability of success that the design meets certain criteria needs to be defined, this is referred to as reliability. There are several methods that can be used to evaluate reliability, such as Monte Carlo simulations (MCS), the first-order reliability method (FORM), and the second-order reliability method (SORM). Details of these methods can be seen in Refs. 2 and 3. In this work the reliability of a $2 \mathrm{D}$ elastostatic boundary element method (BEM) model is evaluated for various levels of uncertainty in design parameters such as the applied stress and the dimensions of the model.

The BEM is a numerical computational method for structural analysis that has found increasing use in the engineering industry over the past few decades since its introduction in the late 1960s. It has proven itself to be an effective tool for structural reliability analysis, with previous applications to $2 \mathrm{D}$ elastostatic structures, ${ }^{1,4}$ fatigue crack growth, ${ }^{5,6}$ and plate bending. ${ }^{7}$ It has developed as an effective alternative to the finite element method (FEM) and has several features that make it an attractive choice. One feature is that the formulations used for the BEM result in a significantly smaller number of equations being obtained than with the FEM, the BEM is also able to obtain a similar level of accuracy to the FEM while using a much coarser mesh. ${ }^{8}$ These advantages allow the BEM to be especially useful for structural reliability analysis. A comprehensive overview of the application of the BEM to structural analysis can be found in Ref. 8 .

There have been several publications relating to structural reliability analysis with the BEM. One notable example is Huang and Aliabadi ${ }^{1}$ where MCS and FORM were used to assess the reliability of a $2 \mathrm{D}$ elastostatics problem involving a rectangular plate with a center circular hole subjected to uniaxial tension. The implicit differentiation method (IDM) was employed to calculate the first-order derivatives for FORM and reliability was evaluated for various levels of uncertainty in material strength and center hole radius. Results showed that MCS and FORM obtained similar results. Huang and Aliabadi ${ }^{6}$ also addressed a more complex problem involving the reliability analysis of $2 \mathrm{D}$ cracked structures using the dual boundary element method (DBEM). ${ }^{8}$ The sensitivity of the stress intensity factors with respect to several random variables, including crack length and fracture toughness, was calculated using the IDM and compared to those obtained from the finite difference method (FDM) with both the DBEM and the FEM and also with MCS. Results showed good agreement between these methods. Lionel and Venturini ${ }^{5}$ built upon this work and assessed the reliability of a problem involving the modeling of crack growth in a 2D perforated panel using MCS and FORM. The failure condition of the structure was based on the number of cycles required for the structure to fail calculated from fatigue crack growth analyses performed using the DBEM. Reliability analyses were carried out using two methods. The first, the direct method, involved the direct coupling of FORM with the DBEM model and approximating the derivatives for FORM using the FDM. The second, the indirect method, involved approximating the response of the DBEM model using a polynomial response 
surface. Results showed that the indirect coupling proved to be less accurate and more computationally expensive than the direct method.

Several recent publications ${ }^{4,7}$ have involved the use of a stochastic spline fictitious boundary element method (SFBEM). The SFBEM is a modified indirect BEM and its use makes it unnecessary to create an explicit expression of the limit state function (LSF), improving the efficiency of the reliability analyses. One example of its use is $\mathrm{Su}$ et $a l^{4}{ }^{4}$ where it was used in conjunction with the advanced first-order second moment (AFOSM) method, a derivative of FORM, to conduct reliability analyses of $2 \mathrm{D}$ elastostatic structures. Results showed that the AFOSM with the SFBEM can obtain good agreement with MCS. Su and $\mathrm{Xu}^{7}$ later applied the SFBEM to a more complex problem involving the structural reliability analysis of Reissner plate bending problems using the AFOSM. The proposed method was validated through comparison with a stochastic FEM method and MCS. Results showed close agreement between the three methods, with the SFBEM proving to be the most efficient, achieving computation times $80 \%$ lower than the stochastic FEM method. As well as the BEM, reliability analyses have also been conducted using the $\mathrm{FEM}^{9,10}$ and experimental data. ${ }^{11}$

Although there have been several publications regarding the use of the BEM for structural reliability analysis,,$^{1,4}$ there have been none involving the use of multifidelity modeling with the BEM, whether for structural reliability analysis or otherwise. The application of multi-fidelity modeling to the topic of structural reliability analysis has also not been investigated thoroughly by the research community, although there have been several instances where surrogate models have been employed successfully. ${ }^{5,10,12}$ The development of reliability analysis formulations incorporating the use of multi-fidelity modeling with the BEM, with its many attractions, is therefore an area of great interest and has the potential to provide significant improvements in efficiency to the employed reliability analysis procedures, especially since assessing the reliability of a structure can be very computationally expensive, particularly for complex problems. With regards to MCS, for relatively simple problems with only two random variables the number of simulations required could be $5 \times 10^{5}$ (Ref. 1), however, for more complex problems, for example with 10 random variables, it could be much higher at $1 \times 10^{8} .{ }^{9}$ By substituting a multi-fidelity model (MFM) for the high-fidelity model (HFM) used with MCS, similar accuracy to the HFM could be achieved at a fraction of the cost. Similar improvements could also be seen with FORM and SORM.

In the context of this work, multi-fidelity modeling, related to surrogate modeling or metamodeling, refers to the process of approximating the response of a HFM over a particular domain. The approximation is created by evaluating the response of the HFM and a low-fidelity model (LFM) at several points, called design points, within this domain. Types of metamodels that have been employed in the past include polynomials, ${ }^{9,10,13,14}$ Kriging, ${ }^{15-17}$ and radial basis neural networks (ANNs). ${ }^{17}$ The aim is to provide a MFM that is of similar accuracy to the HFM over the required 
domain, but at much less computational cost. A recent review of multi-fidelity modeling was undertaken by Simpson et al. ${ }^{18}$

There have been many publications on the topic of multi-fidelity modeling due to its many potential benefits. One notable example is Vitali et al. ${ }^{14}$ where a multifidelity approach was taken toward the calculation of the stress intensity factor in the $0^{\circ}$ plies in a composite stiffened panel with a center crack. Coarse and fine FEM meshes were used as the LFM and HFM, respectively and the ratio and the difference between the stress intensity factors calculated for these models were used to create linear correction response surfaces with the ply thicknesses as independent variables. Results showed that the MFMs were of similar accuracy to the HFM. Sun et al. ${ }^{13}$ used a similar approach for the optimization of a sheet-metal forming process. FEM models were used for the LFM and the HFM with the ratio and difference of the responses between these models being approximated as second-order polynomial correction response surfaces. To avoid calling the LFM for each multi-fidelity approximation, the response of the LFM was approximated using moving least square modeling, reducing computation time. The most effective MFM obtained a low absolute percentage error of $1.06 \%$ with respect to the HFM.

The main objective of this work concerns the development of reliability analysis formulations incorporating the use of multi-fidelity modeling with the BEM. In this work, the computational cost of performing reliability analyses on $2 \mathrm{D}$ elastostatic structures using the BEM through the application of multi-fidelity modeling is investigated. The aim is to use MFMs to obtain similar accuracy to the HFM but at much less computational cost, greatly improving the efficiency of the reliability analysis procedures using MCS, FORM, and SORM. A second objective involves quantifying this improvement in efficiency for each of these three methods, determining the extent to which each of these methods benefit from the application of multi-fidelity modeling.

\section{BEM Models}

The BEM models used in this work consist of a $2 \mathrm{D}$ rectangular plate with a center circular hole, similar to that employed by Huang et al. ${ }^{1}$ The plate has a width $W$ of 1 unit, a length $L$ of 2 units, and a radius $R$ of 0.25 units. The material properties are the Young's modulus, $E=1$ unit, and the Poisson's ratio, $\nu=0.3$ units. The plate is subjected to a uniform far-field remote tensile stress, $\sigma_{T}$, of 1 unit along its top and bottom edges. The plate has roller constraints along the left and right edges and pinned supports at the middle of these edges. A diagram of the plate used in this work can be seen in Fig. 1.

It is necessary to define the failure condition of the plate in order to perform reliability analyses; this can be achieved through the use of a LSF. The LSF in this work is based on the maximum stress in the plate, which occurs as a tangential stress at the edge of the circular hole, and is marked as point $\mathrm{A}$ in Fig. 1. Let, $\mathbf{Z}$ denote a vector of random variables, $\mathbf{Z}=\left(\sigma_{c}, R, \sigma_{T}, \nu, L, W\right)=\left(Z_{1}, Z_{2}, Z_{3}, Z_{4}, Z_{5}, Z_{6}\right)$, and let 


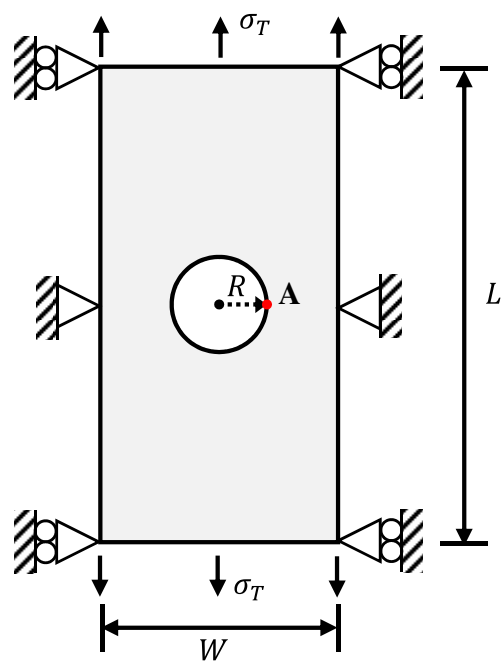

Fig. 1. The rectangular plate used for the BEM models.

$\mathbf{X}(\mathbf{X} \subseteq \mathbf{Z})$ denote the random variables that influence the stress at point $\mathrm{A}$, $\mathbf{X}=\left(R, \sigma_{T}, \nu, L, W\right)$. The LSF, $g(\mathbf{Z})$, in this case is given by:

$$
g(\mathbf{Z})=\sigma_{c}-\sigma_{A}\left(R, \sigma_{T}, \nu, L, W\right)=Z_{1}-\sigma_{A}(\mathbf{X}),
$$

where $\sigma_{c}$ is material strength and $\sigma_{A}$ is the tangential stress at point A. The structure fails when $\sigma_{A}>\sigma_{c}($ or $g(\mathbf{Z})<0)$. The random variables were given the probability distributions as seen in Table 1 . They were assigned parameters based on their expected uncertainty, with $\sigma_{c}$ and $R$ having the most uncertainty in their values. To test the performance of the various models with MCS, FORM, and SORM under varying levels of uncertainty, the coefficient of variation $(\mathrm{COV}=\sigma / \mu$, where $\sigma$ and $\mu$ are the standard deviation and mean, respectively) of the center hole radius, $\mathrm{COV}_{R}$, was varied uniformly in the closed interval $[0,0.1] \Rightarrow\left\{\mathrm{COV}_{R} \in \mathbb{R}: 0 \leq\right.$ $\left.\mathrm{COV}_{R} \leq 0.1\right\}$.

Creating the MFMs requires the definition of a LFM and a HFM. The LFM is a coarse model of the plate as seen in Fig. 1, consisting of 20 quadratic elements, while the HFM is a fine model of 100 elements of the same type. The BEM models can be

Table 1. The six random variables and their probability distribution parameters.

\begin{tabular}{lccccc}
\hline Notation & Symbol & Description & Probability distribution & Mean & Standard deviation \\
\hline$Z_{1}$ & $\sigma_{c}$ & Material strength & Normal & 5.00 & 0.75 \\
$Z_{2}$ & $R$ & Hole radius & Normal & 0.25 & {$[0,0.025]$} \\
$Z_{3}$ & $\sigma_{T}$ & Applied stress & Normal & 1.00 & 0.05 \\
$Z_{4}$ & $\nu$ & Poisson's ratio & Normal & 0.30 & 0.005 \\
$Z_{5}$ & $L$ & Plate length & Normal & 2.00 & 0.025 \\
$Z_{6}$ & $W$ & Plate width & Normal & 1.00 & 0.01 \\
\hline
\end{tabular}



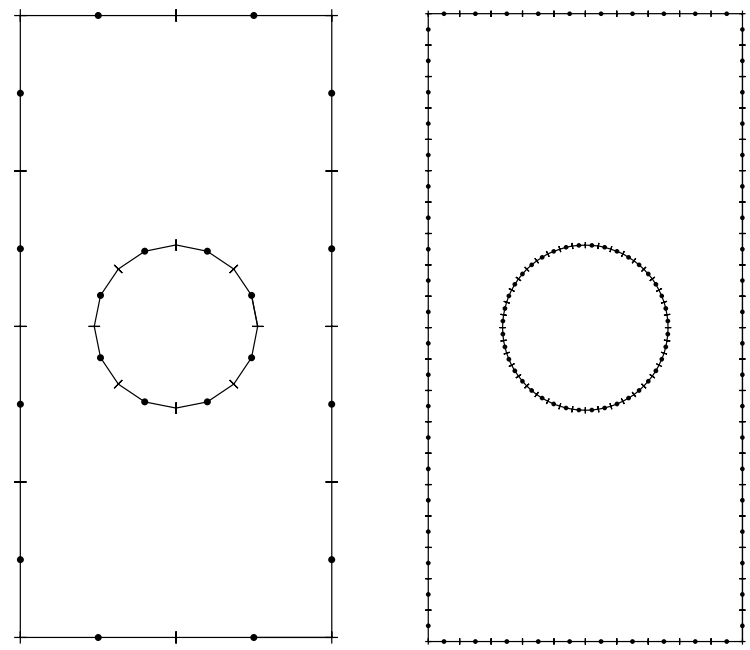

Fig. 2. The LFM (left) with 20 elements and the HFM (right) with 100 elements.

seen in Fig. 2. The ratio of the number of elements on the outer boundary to the number of elements on the hole was kept as $3 / 2$.

\section{Multi-Fidelity Modeling with the BEM}

In this work, response surface methodology (RSM) was used to create response surfaces that would be used to approximate the HFM using surrogate models. The ordinary least squares (OLS) method $^{19}$ is used to approximate the unknown coefficients of the response surfaces. The response surfaces used in this work were second-order polynomial functions, with independent variables $\mathbf{X}$, of the following form:

$$
\begin{aligned}
\hat{y}(\mathbf{X})= & \mathbf{f}(\mathbf{X})^{T} \hat{\boldsymbol{\beta}} \\
= & c_{0}+c_{R} R+c_{\sigma_{T}} \sigma_{T}+c_{\nu} \nu+c_{L} L+c_{W} W+c_{R R} R^{2}+c_{\sigma_{T} \sigma_{T}} \sigma_{T}^{2}+c_{\nu \nu} \nu^{2} \\
& +c_{L L} L^{2}+c_{W W} W^{2}+c_{R \sigma_{T}} R \sigma_{T}+c_{R \nu} R \nu+c_{R L} R L+c_{R W} R W \\
& +c_{\sigma_{T} \nu} \sigma_{T} \nu+c_{\sigma_{T} L} \sigma_{T} L+c_{\sigma_{T} W} \sigma_{T} W+c_{\nu L} \nu L+c_{\nu W} \nu W+c_{L W} L W,
\end{aligned}
$$

where $\hat{y}(\mathbf{X})$ is the response to be approximated and $\mathbf{f}(\mathbf{X})$ is a vector of $p$ terms and is composed of the powers and cross-powers of the independent variables $\mathbf{X}$ :

$$
\begin{gathered}
\mathbf{f}(\mathbf{x})^{\mathbf{T}}=\left(1, R, \sigma_{T}, \nu, L, W, R^{2}, \sigma_{T}^{2}, \nu^{2}, L^{2}, W^{2}, R \sigma_{T}, R \nu,\right. \\
\left.R L, R W, \sigma_{T} \nu, \sigma_{T} L, \sigma_{T} W, \nu L, \nu W, L W\right)
\end{gathered}
$$

$\hat{\boldsymbol{\beta}}$ is a vector of $p$ coefficients:

$$
\begin{aligned}
\hat{\boldsymbol{\beta}}^{T}= & \left(c_{0}, c_{R}, c_{\sigma_{T}}, c_{\nu}, c_{L}, c_{W}, c_{R R}, c_{\sigma_{T} \sigma_{T}}, c_{\nu \nu}, c_{L L}, c_{W W},\right. \\
& \left.c_{R \sigma_{T}}, c_{R \nu}, c_{R L}, c_{R W}, c_{\sigma_{T}}, c_{\sigma_{T} L}, c_{\sigma_{T} W}, c_{\nu L}, c_{\nu W}, c_{L W}\right) .
\end{aligned}
$$


In this work, $p=21$, this includes six linear terms, five squared terms, and 10 mixed terms.

Two types of MFMs were used to approximate the maximum stress in the HFM, $\sigma_{A, \mathrm{HF}}(\mathbf{X})$. The direct type which involved directly calling the LFM, $\sigma_{A, \mathrm{LF}}(\mathbf{X})$, each time a multi-fidelity approximation was required, and the indirect type which involved approximating the LFM as a polynomial response surface, $\sigma_{A, \mathrm{LF}}^{\mathrm{res}}(\mathbf{X})$. In this work, obtaining these MFMs involved obtaining correction response surfaces $\beta^{\text {res }}(\mathbf{X})$ and $\delta^{\text {res }}(\mathbf{X})$, where $\beta$ is the ratio between $\sigma_{A, \mathrm{HF}}(\mathbf{X})$ and $\sigma_{A, \mathrm{LF}}(\mathbf{X})$ and $\delta$ is the difference between $\sigma_{A, \mathrm{HF}}(\mathbf{X})$ and $\sigma_{A, \mathrm{LF}}(\mathbf{X}) \cdot \beta$ and $\delta$ were used in the MFMs instead of derivative-based approximations due to their ability to be applied to larger areas within the design domain and their ability to smooth out noise. ${ }^{14} \beta$ and $\delta$ were calculated at several design points $\mathbf{X}_{i}(i=1,2, \ldots, N)$ to obtain $\beta^{\text {res }}(\mathbf{X})$ and $\delta^{\text {res }}(\mathbf{X})$. The design points were obtained from a Latin hypercube design ${ }^{6,13,14}$ of 30 design points $(N=30)$, requiring a computation time of $18.3 \mathrm{~s}$. In this work, the Latin hypercube design was modified to improve its application to reliability analysis; the design points were obtained by uniformly sampling from each random variable's cumulative distribution function (CDF) between the probabilities $2.5 \%$ and $97.5 \%$. This ensured that the design points were highly concentrated in the highly-likely areas of each variable's domain, and less concentrated in the less-likely areas. The response surfaces were created with the parameters in Table 1 with $\mathrm{COV}_{R}=0.05$ to provide good accuracy over the entire range of $\mathrm{COV}_{R}$ values investigated. A fifth surrogate model for the HFM was obtained by approximating $\sigma_{A, \mathrm{HF}}(\mathbf{X})$ as a response surface, $\sigma_{A, \mathrm{HF}}^{\text {res }}(\mathbf{X})$. The details of the seven models are shown in Table 2.

\subsection{Model performance evaluation}

To test the robustness of these response surfaces, they were run with the design points from a second Latin hyper cube design, the "test" dataset. This dataset consisted of 100 design points sampled from each variable's CDF between a wider range of probabilities, $0.5 \%$ to $99.5 \%$. The "design" dataset consisted of the 30 design points used to create each response surface. Various statistical parameters evaluated for the response surfaces $\beta^{\mathrm{res}}(\mathbf{X}), \delta^{\mathrm{res}}(\mathbf{X}), \sigma_{A, \mathrm{LF}}^{\mathrm{res}}(\mathbf{X})$, and $\sigma_{A, \mathrm{HF}}^{\text {res }}(\mathbf{X})$ with the design and test datasets can be seen in Table 3 . These were the coefficient of determination

Table 2. The $\sigma_{A}$ approximations and LSFs of the seven models investigated.

\begin{tabular}{|c|c|c|}
\hline Model & $\sigma_{A}$ approximation & LSF \\
\hline MF1 & $\sigma_{A, \mathrm{MF} 1}(\mathbf{X})=\beta^{\mathrm{res}}(\mathbf{X}) \sigma_{A, \mathrm{LF}}(\mathbf{X})$ & $g_{\mathrm{MF} 1}(\mathbf{Z})=Z_{1}-\sigma_{A, \mathrm{MF} 1}(\mathbf{X})$ \\
\hline MF2 & $\sigma_{A, \mathrm{MF} 2}(\mathbf{X})=\delta^{\mathrm{res}}(\mathbf{X})+\sigma_{A, \mathrm{LF}}(\mathbf{X})$ & $g_{\mathrm{MF} 2}(\mathbf{Z})=Z_{1}-\sigma_{A, \mathrm{MF} 2}(\mathbf{X})$ \\
\hline MF3 & $\sigma_{A, \mathrm{MF} 3}(\mathbf{X})=\beta^{\mathrm{res}}(\mathbf{X}) \sigma_{A, \mathrm{LF}}^{\mathrm{res}}(\mathbf{X})$ & $g_{\mathrm{MF} 3}(\mathbf{Z})=Z_{1}-\sigma_{A, \mathrm{MF} 3}(\mathbf{X})$ \\
\hline MF4 & $\sigma_{A, \mathrm{MF} 4}(\mathbf{X})=\delta^{\mathrm{res}}(\mathbf{X})+\sigma_{A, \mathrm{LF}}^{\mathrm{res}}(\mathbf{X})$ & $g_{\mathrm{MF} 4}(\mathbf{Z})=Z_{1}-\sigma_{A, \mathrm{MF} 4}(\mathbf{X})$ \\
\hline $\mathrm{HFM}^{\text {res }}$ & $\sigma_{A, \mathrm{HF}}^{\mathrm{res}}(\mathbf{X})$ & $g_{\mathrm{HF}, \mathrm{res}}(\mathbf{Z})=Z_{1}-\sigma_{A, \mathrm{HF}}^{\mathrm{res}}(\mathbf{X})$ \\
\hline LFM & $\sigma_{A, \mathrm{LF}}(\mathbf{X})=\sigma_{A, E 20}(\mathbf{X})$ & $g_{\mathrm{LF}}(\mathbf{Z})=Z_{1}-\sigma_{A, \mathrm{LF}}(\mathbf{X})$ \\
\hline HFM & $\sigma_{A, \mathrm{HF}}(\mathbf{X})=\sigma_{A, E 100}(\mathbf{X})$ & $g_{\mathrm{HF}}(\mathbf{Z})=Z_{1}-\sigma_{A, \mathrm{HF}}(\mathbf{X})$ \\
\hline
\end{tabular}


Table 3. Various statistical parameters evaluated for the response surfaces $\beta^{\text {res }}(\mathbf{X})$, $\delta^{\mathrm{res}}(\mathbf{X}), \sigma_{A, \mathrm{LF}}^{\mathrm{res}}(\mathbf{X})$, and $\sigma_{A, \mathrm{HF}}^{\text {res }}(\mathbf{X})$ with the design $(D)$ and test $(T)$ datasets.

\begin{tabular}{lcccc}
\hline Statistical parameter & $\beta^{\text {res }}(\mathbf{X})$ & $\delta^{\text {res }}(\mathbf{X})$ & $\sigma_{A, \mathrm{LF}}^{\text {res }}(\mathbf{X})$ & $\sigma_{A, \mathrm{HF}}^{\text {res }}(\mathbf{X})$ \\
\hline$R_{D}^{2}$ & 1.0000 & 1.0000 & 1.0000 & 1.0000 \\
$R_{\text {adj }, D}^{2}$ & 1.0000 & 1.0000 & 1.0000 & 1.0000 \\
$\mathrm{SE}_{D}$ & $9.54 \times 10^{-5}$ & $3.00 \times 10^{-4}$ & $2.67 \times 10^{-2}$ & $2.25 \times 10^{-2}$ \\
$\mathrm{MAPE}_{D}(\%)$ & $1.16 \times 10^{-4}$ & $5.78 \times 10^{-1}$ & $4.52 \times 10^{-3}$ & $2.27 \times 10^{-3}$ \\
$R_{T}^{2}$ & 0.9999 & 0.9992 & 1.0000 & 1.0000 \\
$R_{\text {adj }, T}^{2}$ & 0.9998 & 0.9989 & 1.0000 & 1.0000 \\
$\mathrm{SE}_{T}$ & $6.04 \times 10^{-5}$ & $1.88 \times 10^{-4}$ & $1.69 \times 10^{-2}$ & $1.57 \times 10^{-2}$ \\
$\mathrm{MAPE}_{T}(\%)$ & $4.03 \times 10^{-4}$ & 6.57 & $1.53 \times 10^{-2}$ & $1.14 \times 10^{-2}$ \\
\hline
\end{tabular}

$R^{2}$, the adjusted coefficient of determination $R_{\text {adj }}^{2}$, the standard error (SE), and the mean absolute percentage error (MAPE).

The data in Table 3 suggests that the created response surfaces are able to provide highly accurate approximations for the responses of the LFM and the HFM over the required domain. $\beta^{\text {res }}(\mathbf{X})$ proved to be more accurate than $\delta^{\text {res }}(\mathbf{X})$, this suggests that MFMs using $\beta^{\text {res }}(\mathbf{X})$ could prove to be more accurate.

To determine the performance of these surrogate models they were compared to the performance of several BEM models of a range of fidelities. $\sigma_{A}$ was calculated at the mean values of the parameters seen in Table 1 for each of the models. The average computation time for each was calculated over 1000 runs. The results are shown in Table 4.

It can be seen from Table 4 that the indirect MFMs utilizing $\sigma_{A, \mathrm{LF}}^{\mathrm{res}}$ have similar accuracy to $\sigma_{A, \mathrm{HF}}^{\text {res }}$. The most accurate MFMs involved directly calling the LFM, and of those the most accurate approximations were those that utilized $\beta^{\text {res }}$, while those

Table 4. MAPE (\%) of the various surrogate models and BEM models with respect to the HFM. Also shown are the average CPU wall-times over 1000 runs for each model. " $X$ " in $\sigma_{A, E X}$ denotes the number of elements in a BEM model.

\begin{tabular}{lccc}
\hline Model type & Model & MAPE $(\%)$ & Average computation time per run (ms) \\
\hline Direct MFMs & $\sigma_{A, \mathrm{MF} 1}$ & $6.38 \times 10^{-4}$ & 142 \\
& $\sigma_{A, \mathrm{MF} 2}$ & $8.43 \times 10^{-4}$ & 138 \\
Indirect MFMs & $\sigma_{A, \mathrm{MF} 3}$ & $1.57 \times 10^{-2}$ & 0.402 \\
& $\sigma_{A, \mathrm{MF} 4}$ & $1.62 \times 10^{-2}$ & 0.412 \\
HFM response surface & $\sigma_{A, \mathrm{HF}}^{\text {res }}$ & $1.14 \times 10^{-2}$ & 0.247 \\
BEM models & $\sigma_{A, E 10}$ & 3.40 & 66 \\
& $\sigma_{A, E 20}(\mathrm{LFM})$ & $6.71 \times 10^{-2}$ & 110 \\
& $\sigma_{A, E 40}$ & $4.88 \times 10^{-2}$ & 155 \\
& $\sigma_{A, E 60}$ & $1.15 \times 10^{-2}$ & 255 \\
& $\sigma_{A, E 80}$ & $3.07 \times 10^{-3}$ & 328 \\
& $\sigma_{A, E 100}(\mathrm{HFM})$ & 0.00 & 499 \\
\hline
\end{tabular}


utilizing $\delta^{\text {res }}$ proved to be less accurate. This agrees well with observations made of the data presented previously in Table 3 . The best approximation was given by the multi-fidelity model MF1, which achieved the lowest MAPE of $6.38 \times 10^{-4 \%}$. This model is 4.81 times more accurate than the 80 -element mesh, $\sigma_{A, E 80}$, while also having a CPU wall-time 2.65 times less. $\sigma_{A, \mathrm{HF}}^{\mathrm{res}}$ proved to be the least accurate approximation, achieving similar accuracy to the 60 -element mesh, $\sigma_{A, E 60}$, but with a computation time about 1032 times less.

Results suggest that MF1 and MF2 both act as good substitutes for the HFM, achieving very similar levels of accuracy and with computation times similar to that of the LFM. On the other hand, the indirect models, MF3 and MF4, as well as $\mathrm{HFM}^{\text {res }}$ proved to be less accurate, but showed much lower computation times.

\section{Multi-Fidelity Modeling-Based Structural Reliability Analysis}

In this section, multi-fidelity modeling is used in combination with reliability analyses performed using MCS, FORM, and SORM. The models shown in Table 2 are investigated, this includes the two BEM models, the LFM and the HFM, the four MFMs (MF1-MF4), and the response surface approximation of the high-fidelity BEM model, HFM $^{\text {res }}$.

The reliability, $P_{R}$, of a structure can be determined by evaluating the following integral:

$$
P_{R}=1-P_{F}=P\{g(\mathbf{Z})>0\}=\int_{g(\mathbf{Z})>0} f_{\mathbf{Z}}(\mathbf{Z}) d \mathbf{Z},
$$

where $f_{\mathbf{Z}}(\mathbf{Z})$ is the joint probability density function of $\mathbf{Z}$, and $P_{F}$ is the probability of failure. $P_{F}$ and $P_{R}$ involve integrating $f_{\mathbf{Z}}(\mathbf{Z})$ over the regions defined by $g(\mathbf{Z})<0$ (the failure region) and $g(\mathbf{Z})>0$ (the safe region), respectively. All the random variables are assumed to be mutually independent. The direct evaluation of the above integral is usually very difficult since it can be multidimensional if many random variables are involved. The integration boundary $g(\mathbf{Z})=0$ can also be multidimensional and is usually a nonlinear function. The LSF, $g(\mathbf{Z})$, may also be a black-box model (in this case a BEM model) and so it may be computationally expensive to evaluate.

There are several methods that can be used to evaluate this integral; MCS, which involves sampling from each random variable's distribution, and FORM and SORM, which attempt to simplify the integration.

\subsection{Monte Carlo simulation (MCS)}

MCS involves randomly sampling from known probability distributions to determine an unknown probability distribution. ${ }^{3}$ If $\mathbf{Z}=\left(Z_{1}, Z_{2}, \ldots, Z_{n}\right)$ is a vector of known random variables, and if $Y=h(\mathbf{Z})$, where $Y$ is a random variable with an unknown probability distribution and $h$ is some process or function, then we can randomly 
sample the variables in $\mathbf{Z}$ per their probability distributions, input them into $h$ and obtain a value for $Y$. This is repeated many times until a histogram can be created of $Y$, allowing us to estimate its probability distribution.

MCS can be used to evaluate the LSFs in Table 2 to obtain the reliability of the structure with the random variables $\mathbf{Z}=\left(Z_{1}, Z_{2}, \ldots, Z_{6}\right)=\left(\sigma_{c}, R, \sigma_{T}, \nu, L, W\right)$ described in Table 1 . For each simulation, the variables in $\mathbf{Z}$ are randomly sampled from their probability distributions, with the samples of the last five variables $\left(R, \sigma_{T}, \nu, L, W\right)$ being used as inputs to a BEM model to evaluate $\sigma_{A}$. If $g(\mathbf{Z})<0$ $\left(\sigma_{A}\left(R, \sigma_{T}, \nu, L, W\right)>\sigma_{c}\right)$ for a simulation, then the structure is assumed to have failed. After a certain number of simulations have been carried out, $N_{\text {MCS }}$, the total number of simulations in which the structure failed, $N_{F}$, can be determined and the failure probability $P_{F}$ can be calculated as:

$$
P_{F}=\frac{N_{F}}{N_{\mathrm{MCS}}} .
$$

Reliability can be calculated as:

$$
P_{R}=1-P_{F} .
$$

Because MCS is a simple brute-force sampling method, it can be used as a benchmark to validate the results obtained from the LSF approximation methods, FORM and SORM.

\subsection{First-order reliability method (FORM)}

FORM used in this work refers to the AFOSM method for nonlinear LSFs. AFOSM is an improvement over previous FORM methods such as the mean value first-order second-moment (MVFOSM) method and the Hasofer-Lind (HL) AFOSM method in that it can be used with better accuracy with non-normal random variables and nonlinear LSFs. ${ }^{2}$

There are two steps that FORM takes to make the integration in Eq. (5) more manageable. The first step involves simplifying $f_{\mathbf{Z}}(\mathbf{Z})$ such that its contours are more regular and symmetric; this is achieved by transforming the random variables from Z-space into U-space, the standard normal space. The second step involves approximating the integration boundary $g(\mathbf{U})=0$ as a first-order Taylor expansion:

$$
g(\mathbf{U}) \approx g\left(\mathbf{U}^{*}\right)+\nabla g\left(\mathbf{U}^{*}\right)\left(\mathbf{U}-\mathbf{U}^{*}\right)^{T},
$$

where $\mathbf{U}$ is created by transforming $\mathbf{Z}$ into $\mathbf{U}$-space, $\mathbf{U}^{*}$ is the expansion point, and $\nabla g\left(\mathbf{U}^{*}\right)$ is:

$$
\nabla g\left(\mathbf{U}^{*}\right)=\left.\left(\frac{\partial g(\mathbf{U})}{\partial U_{1}}, \frac{\partial g(\mathbf{U})}{\partial U_{2}}, \ldots, \frac{\partial g(\mathbf{U})}{\partial U_{n}}\right)\right|_{\mathbf{U}^{*}}
$$

To minimize the accuracy lost by this approximation, it is necessary to expand $g(\mathbf{U})$ at the point $\mathbf{U}^{*}$ that contributes the most to the integrations as seen in Eq. (5) and so it will be the point that corresponds to the highest probability density. This 
point is termed as the most probable point (MPP $)^{3}$ and is the point along the integration boundary $g(\mathbf{U})=0$ that is closest to the origin of U-space. The distance between the MPP and the origin of $\mathrm{U}$-space is termed the reliability index $\beta . \beta$ is related to the probability of failure $P_{F}$ and reliability $P_{R}$ in the following manner:

$$
P_{R}=1-P_{F}=1-\Phi(-\beta)=\Phi(\beta)
$$

where $\Phi$ denotes the standard normal CDF. In this work, a Newton-Raphson type recursive algorithm developed by Rackwitz and Fiessler ${ }^{20}$ is used to determine the MPP.

\subsection{Second-order reliability method (SORM)}

The SORM involves approximating $g(\mathbf{U})=0$ as a second-order Taylor expansion:

$$
g(\mathbf{U}) \approx g\left(\mathbf{U}^{*}\right)+\nabla g\left(\mathbf{U}^{*}\right)\left(\mathbf{U}-\mathbf{U}^{*}\right)^{T}+\frac{1}{2}\left(\mathbf{U}-\mathbf{U}^{*}\right) \mathbf{H}\left(\mathbf{U}^{*}\right)\left(\mathbf{U}-\mathbf{U}^{*}\right)^{T},
$$

where $H\left(\mathbf{U}^{*}\right)$ is the Hessian matrix evaluated at $\mathbf{U}^{*}$ and contains the second-order derivatives of the LSF with respect to the random variables in U-space. Since SORM is of a higher-order than FORM, it is expected that it will more accurately approximate nonlinear LSFs, such as those involved in this work. The SORM used in this work is Breitung's asymptotic approximation ${ }^{21}$ :

$$
P_{F}=P(g(\mathbf{X})<0)=\Phi\left(-\beta_{\mathrm{FORM}}\right) \prod_{i=1}^{n-1}\left(1+\beta_{\mathrm{FORM}} \kappa_{i}\right)^{-1 / 2}
$$

where $\beta_{\text {FORM }}$ is the converged value of the reliability index calculated from FORM and $\kappa_{i}(i=1, \ldots, n-1)$ are the principal curvatures of the LSF at the converged MPP location $\mathbf{U}^{*}$ from FORM. This approximation is accurate only for large values of $\beta$, which is the case for practical high-reliability problems. ${ }^{2,22}$

\subsection{Numerical example}

FORM and SORM require the derivatives of the LSF under investigation, which can be directly calculated for the response surfaces $\beta^{\text {res }}, \delta^{\text {res }}, \sigma_{A, \mathrm{LF}}^{\mathrm{res}}$, and $\sigma_{A, \mathrm{HF}}^{\mathrm{res}}$. The calculation of the derivatives of $\sigma_{A, \mathrm{LF}}$ and $\sigma_{A, \mathrm{HF}}$ is more complex as they require the differentiation of the response of the BEM models. Methods used in the past include the $\mathrm{IDM}^{1,23}$ and the FDM. ${ }^{5}$ In this work, the FDM is used with the first-order forward scheme and second-order central difference scheme. The step size in U-space, $\Delta U$, was set as $1 \times 10^{-5}$ for FORM and $1 \times 10^{-3}$ for SORM as these were found to provide accurate results. The first-order derivatives were evaluated in the following manner (for $i \neq 1$ ):

$$
\frac{\partial g(\mathbf{U})}{\partial U_{i}}=\sigma_{z_{i}} \frac{\partial g(\mathbf{Z})}{\partial Z_{i}}=\sigma_{z_{i}}\left(-\frac{\sigma_{A}\left(Z_{i}+\Delta Z_{i}, \mathbf{Z}_{-i}\right)-\sigma_{A}\left(Z_{i}, \mathbf{Z}_{-i}\right)}{\Delta Z_{i}}\right) .
$$


For $i=1$ :

$$
\frac{\partial g(\mathbf{U})}{\partial U_{1}}=\sigma_{z_{1}} \frac{\partial g(\mathbf{Z})}{\partial Z_{1}}=\sigma_{z_{1}}
$$

where $\sigma_{z_{i}}$ is the standard deviation of the $i$ 'th random variable and $\Delta Z_{i}=\sigma_{z_{i}} \Delta U$. $\mathbf{Z}_{-i}$ is a vector that includes all the random variables in $\mathbf{Z}$ that influence $\sigma_{A}$ but excludes $Z_{i}$. The second-order derivatives were calculated as (for $i, j \neq 1$ and $i=j$ ):

$$
\frac{\partial^{2} g(\mathbf{U})}{\partial U_{i}^{2}}=\sigma_{z_{i}}^{2}\left(-\frac{\sigma_{A}\left(Z_{i}+\Delta Z_{i}, \mathbf{Z}_{-i}\right)-2 \sigma_{A}\left(Z_{i}, \mathbf{Z}_{-i}\right)+\sigma_{A}\left(Z_{i}-\Delta Z_{i}, \mathbf{Z}_{-i}\right)}{\left(\Delta Z_{i}\right)^{2}}\right)
$$

For $i, j \neq 1$ and $i \neq j$ :

$$
\frac{\partial^{2} g(\mathbf{U})}{\partial U_{i} \partial U_{j}}=\sigma_{z_{i}} \sigma_{z_{j}}\left(-\frac{\sigma_{A,(i+1, j+1)}-\sigma_{A,(i+1, j-1)}-\sigma_{A,(i-1, j+1)}+\sigma_{A,(i-1, j-1)}}{4 \Delta Z_{i} \Delta Z_{j}}\right),
$$

where

$$
\begin{aligned}
& \sigma_{A,(i+1, j+1)}=\sigma_{A}\left(Z_{i}+\Delta Z_{i}, Z_{j}+\Delta Z_{j}, \mathbf{Z}_{-i,-j}\right) \\
& \sigma_{A,(i+1, j-1)}=\sigma_{A}\left(Z_{i}+\Delta Z_{i}, Z_{j}-\Delta Z_{j}, \mathbf{Z}_{-i,-j}\right) \\
& \sigma_{A,(i-1, j+1)}=\sigma_{A}\left(Z_{i}-\Delta Z_{i}, Z_{j}+\Delta Z_{j}, \mathbf{Z}_{-i,-j}\right), \\
& \sigma_{A,(i-1, j-1)}=\sigma_{A}\left(Z_{i}-\Delta Z_{i}, Z_{j}-\Delta Z_{j}, \mathbf{Z}_{-i,-j}\right),
\end{aligned}
$$

where $\mathbf{Z}_{-i,-j}$ is a vector that includes all the random variables in $\mathbf{Z}$ that influence $\sigma_{A}$ but excludes $Z_{i}$ and $Z_{j}$. For $i=1$ or $j=1$ :

$$
\frac{\partial^{2} g(\mathbf{U})}{\partial U_{i} \partial U_{j}}=0
$$

The reliability of each of the seven models was evaluated using FORM and SORM at 101 uniformly-spaced values of $\mathrm{COV}_{R}$ in the closed interval [0,0.1]. For MCS, 11 uniformly-spaced values were investigated. A total of $2.2 \times 10^{9}$ MCS simulations were carried out for each of the seven models, $2 \times 10^{8}$ for each of the 11 reliability indices corresponding to the 11 values of $\mathrm{COV}_{R}$.

\subsection{Results and discussion}

Figure 3 shows the reliability indices obtained from MCS, FORM, and SORM for the LFM and the HFM. It can be seen from this figure that SORM follows MCS much more closely than FORM, suggesting that SORM provides a much more accurate approximation for the failure domain than FORM. This is because SORM approximates each of these nonlinear LSFs in Table 2 using a second-order Taylor expansion, taking into account the curvature of the LSFs, therefore providing a more accurate evaluation of the failure domain. The consequence of this is that SORM is more computationally expensive than FORM, as can be seen in Fig. 4. For a low level of uncertainty $\left(\mathrm{COV}_{R}=0\right)$, the MAPE between MCS and FORM for the HFM is 


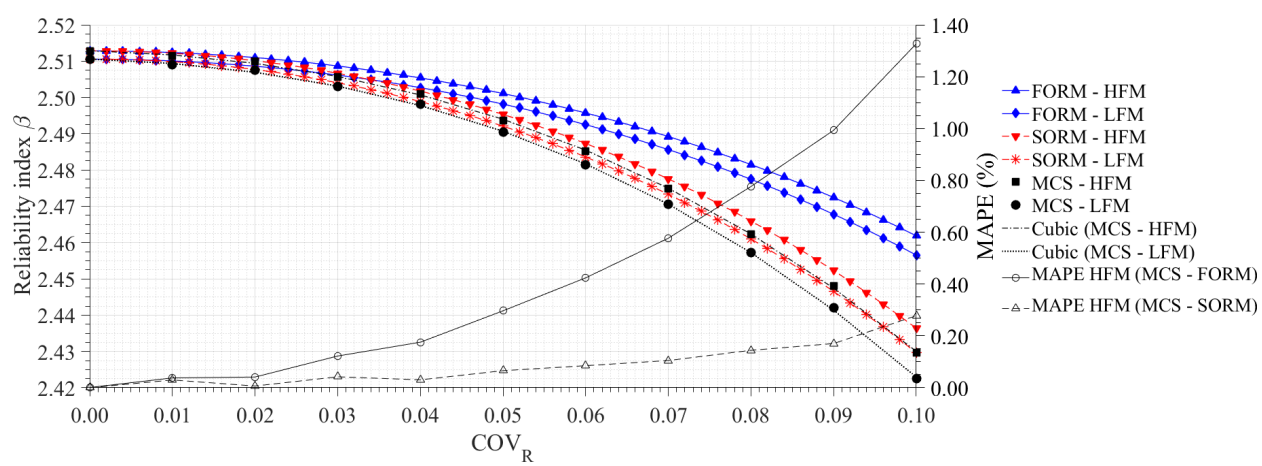

Fig. 3. Reliability indices calculated using MCS, FORM, and SORM for the LFM and the HFM. Also shown is the MAPE between MCS and FORM and MCS and SORM for the HFM at each $\operatorname{COV}_{R}$ value.

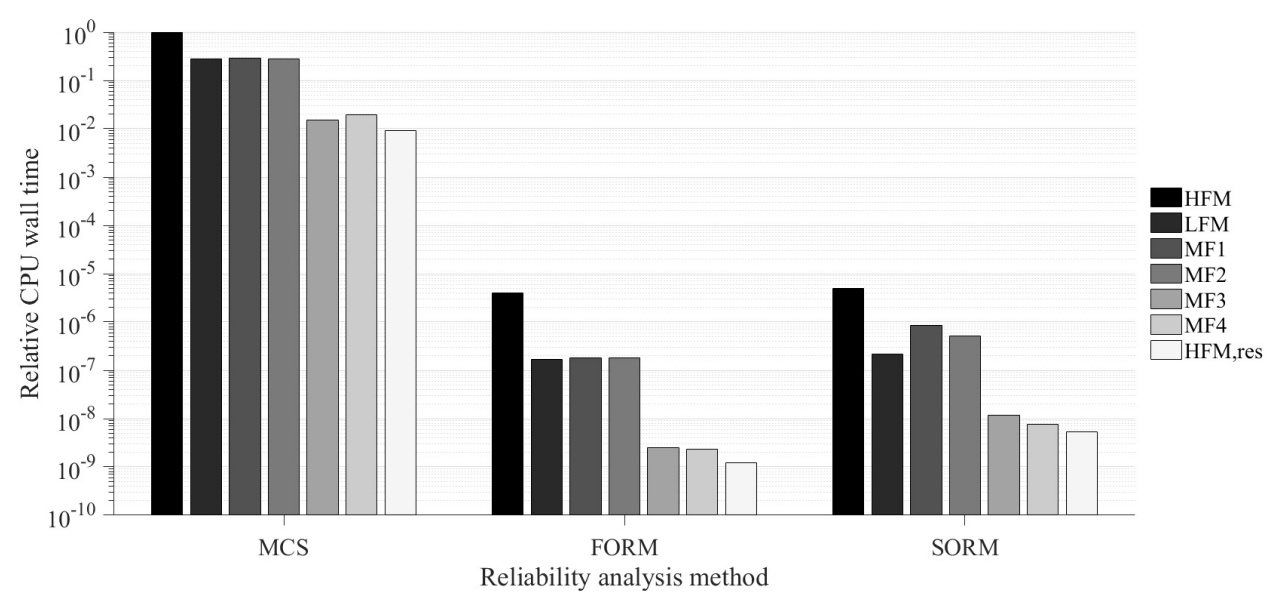

Fig. 4. The ratio of the average CPU wall-time of each model for MCS, FORM, and SORM to the average CPU wall-time of the HFM for MCS. Average CPU wall-time is defined as the time required to obtain a reliability index for a model.

$9.80 \times 10^{-4 \%}$ while it is smaller at $2.13 \times 10^{-4 \%}$ for SORM. In Fig. 4 and Table 5, it can be seen that FORM is on average $3.41 \times 10^{6}$ times faster than MCS, while SORM is on average $1.05 \times 10^{6}$ times faster. SORM is also on average 4.98 times more accurate than FORM, but has a computation time that is on average 3.20 times greater. A significant portion of this time is used to evaluate the entries of the Hessian matrix $H\left(\mathbf{U}^{*}\right)$ which requires a total of $2 n^{2}$ calls to a BEM model, where $n$ is the number of random variables being investigated. Because of the symmetry of $H\left(\mathbf{U}^{*}\right)$, it is only necessary to directly evaluate half of its non-diagonal entries. FORM, by comparison, requires $n+1$ calls to the BEM model for each iteration of the MPP search algorithm. Given that in this work $n=6$ and that on average the MPP search algorithm required six iterations, FORM required a total of 42 calls to 
Table 5. MAPE (\%) of the various models with respect to the HFM and the average CPU wall-time (s) required to obtain a reliability index value. A total of $2 \times 10^{8}$ MCS simulations were required for each data point/reliability index value for each model.

\begin{tabular}{|c|c|c|c|c|c|c|}
\hline \multirow[b]{2}{*}{ Model } & \multicolumn{3}{|c|}{ Average MAPE (\%) } & \multicolumn{3}{|c|}{ Average computation time (s) per data point } \\
\hline & MCS & FORM & SORM & MCS & FORM & SORM \\
\hline LFM & $1.56 \times 10^{-1}$ & $1.29 \times 10^{-1}$ & $1.48 \times 10^{-1}$ & $9.06 \times 10^{5}$ & $5.59 \times 10^{-1}$ & $7.16 \times 10^{-1}$ \\
\hline MF1 & $5.82 \times 10^{-4}$ & $1.27 \times 10^{-4}$ & $7.71 \times 10^{-4}$ & $9.79 \times 10^{5}$ & $6.11 \times 10^{-1}$ & 2.83 \\
\hline MF2 & $2.81 \times 10^{-3}$ & $5.02 \times 10^{-4}$ & $1.50 \times 10^{-3}$ & $9.45 \times 10^{5}$ & $6.09 \times 10^{-1}$ & 1.69 \\
\hline MF3 & $4.60 \times 10^{-2}$ & $1.02 \times 10^{-2}$ & $2.66 \times 10^{-2}$ & $4.26 \times 10^{4}$ & $8.21 \times 10^{-3}$ & $3.85 \times 10^{-2}$ \\
\hline MF4 & $4.47 \times 10^{-2}$ & $1.02 \times 10^{-2}$ & $2.54 \times 10^{-2}$ & $8.08 \times 10^{4}$ & $7.84 \times 10^{-3}$ & $2.59 \times 10^{-2}$ \\
\hline HFM $^{\text {res }}$ & $7.36 \times 10^{-2}$ & $7.34 \times 10^{-3}$ & $4.53 \times 10^{-2}$ & $1.35 \times 10^{4}$ & $4.00 \times 10^{-3}$ & $1.77 \times 10^{-2}$ \\
\hline HFM & 0.00 & 0.00 & 0.00 & $3.26 \times 10^{6}$ & $1.31 \times 10^{1}$ & $1.68 \times 10^{1}$ \\
\hline
\end{tabular}

the BEM model on average to obtain a reliability index value. SORM required 72 for the evaluation of $H\left(\mathbf{U}^{*}\right)$, and since SORM relies on the results from FORM, it therefore required a total of 114 calls on average to obtain a reliability index value. Both FORM and SORM become less accurate as $\mathrm{COV}_{R}$ increases, this is due to the increasing level of uncertainty.

Given the data shown in Table 4 it was expected that the direct MFMs (MF1 and MF2) would prove to be the most accurate surrogate models for reliability analysis. This seems to be the case when looking at Figs. 5-8 where they are shown to be significantly superior in accuracy to the LFM while also being of similar computational cost, suggesting that they could act as very good substitutes for the HFM. The indirect MFMs (MF3 and MF4) and the response surface approximation of the HFM, $\mathrm{HFM}^{\text {res }}$, by comparison were shown to be less accurate than the direct models but significantly faster in terms of computation time, achieving wall-times that were around 10-20 times less than the direct MFMs for MCS, with HFM ${ }^{\text {res }}$ proving to be the fastest at 70 times less. Despite this, the accuracy of the indirect MFMs is similar to that of the direct MFMs for low levels of uncertainty $\left(\mathrm{COV}_{R}<0.05\right)$, but worsens

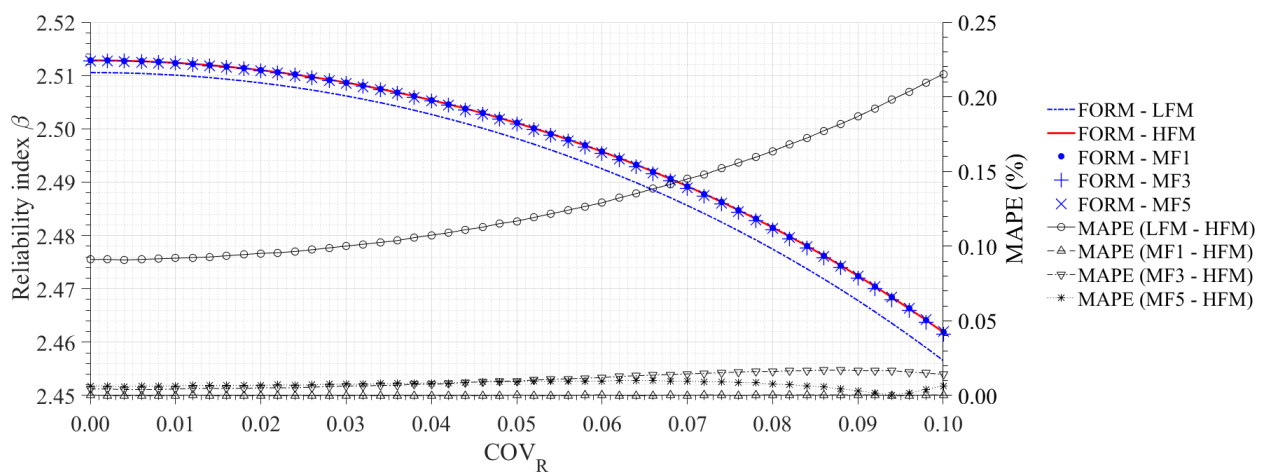

Fig. 5. Reliability indices calculated using FORM for the models LFM, HFM, MF1, MF3, and MF5. Also shown is the MAPE of the models with respect to the HFM with FORM at each $\mathrm{COV}_{R}$ value. 


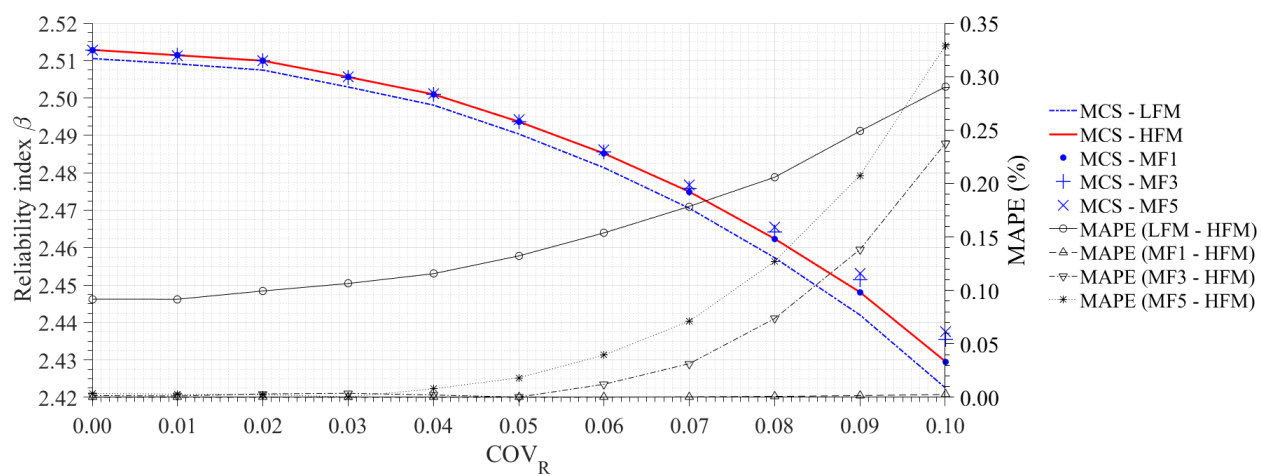

Fig. 6. Reliability indices calculated using SORM for the models LFM, HFM, MF1, MF3, and MF5. Also shown is the MAPE of the models with respect to the HFM with SORM at each $\mathrm{COV}_{R}$ value.

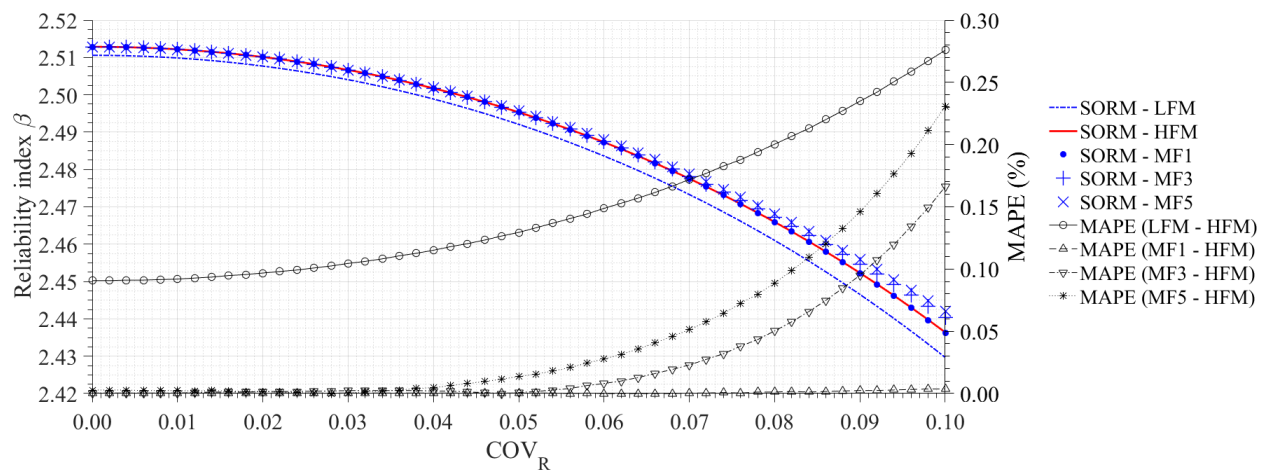

Fig. 7. Reliability indices calculated using MCS for the models LFM, HFM, MF1, MF3, and MF5. Also shown is the MAPE of the models with respect to the HFM with MCS at each $\mathrm{COV}_{R}$ value.

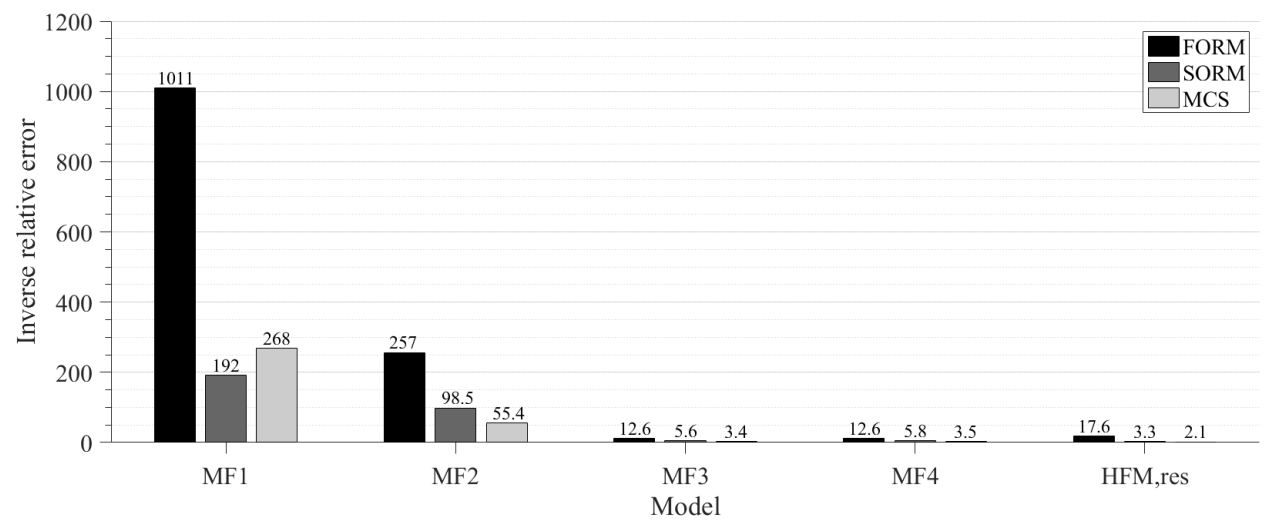

Fig. 8. The inverse of the ratio of each model's average MAPE to the average MAPE of the LFM for MCS, FORM, and SORM. 
after this. This is due to the fact that the response surfaces were designed at $\mathrm{COV}_{R}=$ 0.05 in order to minimize the average error over the range of $\mathrm{COV}_{R}$ values investigated. A method to mitigate this would be to design a set of response surfaces for each $\mathrm{COV}_{R}$ under investigation. The most accurate MFM proved to be MF1, which provided the greatest accuracy improvement over the LFM for MCS, FORM, and SORM. This was expected from the results shown previously in Table 4. Although it provides very high accuracy over the entire range of $\mathrm{COV}_{R}$ values investigated, achieving a very small MAPE for MCS with respect to the HFM of $2.7 \times 10^{-3 \%}$ for $\mathrm{COV}_{R}=0.10$, it is most effective when used for low levels of uncertainty. MF1 provided very similar computation times to the LFM, being only $7.95 \%$ slower for MCS and $9.27 \%$ for FORM. MF1 is significantly slower than the LFM for SORM, providing computation times that are $295.47 \%$ greater, this is because the second derivative of its LSF contains six terms and requires three calls to the LFM in the case where $i=j$ and in the case $i \neq j 8$ terms need to be evaluated and six calls to the LFM are required. In contrast, the second derivative of the LFM's LSF only contains one term that requires two calls for $i=j$ or four calls for $i \neq j$.

From Fig. 5, we can see that all the MFMs perform similarly well with FORM. This can also be seen from the sensitivities in Table 6 which are used by FORM. These sensitivities quantify the influence that a change in each of the random variables in $\mathbf{X}$ has on $\sigma_{A}$. It is also worth mentioning that the sensitivities are in-line with what is expected, with $R$ and $\sigma_{T}$ having the largest influence on $\sigma_{A}$, and $\nu$ and $L$ having the least amount of influence. The MFMs with SORM in Fig. 6, by comparison, perform very differently, with the indirect models proving to be less accurate than the direct models, especially at high levels of uncertainty $\left(\operatorname{COV}_{R}>0.05\right)$. This can be explained by the fact that the response surfaces used in this work are second-order polynomials, their second-order derivatives are therefore constant. The effect of this is maximized in the case of the indirect models since they are purely constructed of response surfaces. A means of mitigating this would be to use higherorder polynomials, possibly third or fourth order, at the expense of increased computation time. From Fig. 8, we can see that the reliability analysis method that showed the greatest improvement in accuracy over the LFM when used in conjunction with the MFMs was FORM, which experienced a 1011 times reduction in

Table 6. Sensitivities of $\sigma_{A}$ for the seven models investigated with respect to the random variables $\mathbf{Z}$ evaluated at their mean values.

\begin{tabular}{lccccccc}
\hline \multicolumn{7}{c}{ Sensitivity $\delta \sigma_{A} / \delta Z_{i}$} \\
\cline { 2 - 7 } $\mathbf{Z}_{i}$ & LFM & MF1 & MF2 & MF3 & MF4 & HFM $^{\text {res }}$ & HFM \\
\hline$R$ & 5.85 & 5.69 & 5.80 & 5.73 & 5.73 & 5.72 & 5.70 \\
$\sigma$ & 3.08 & 3.08 & 3.08 & 3.08 & 3.08 & 3.08 & 3.08 \\
$\nu$ & $-8.19 \times 10^{-1}$ & $-7.86 \times 10^{-1}$ & $-8.08 \times 10^{-1}$ & $-7.64 \times 10^{-1}$ & $-7.62 \times 10^{-1}$ & $-7.74 \times 10^{-1}$ & $-7.85 \times 10^{-1}$ \\
$L$ & $-1.93 \times 10^{-3}$ & $-1.42 \times 10^{-3}$ & $-1.76 \times 10^{-3}$ & $-3.77 \times 10^{-3}$ & $-3.75 \times 10^{-3}$ & $-1.01 \times 10^{-3}$ & $-1.37 \times 10^{-3}$ \\
$W$ & -1.46 & -1.42 & -1.45 & -1.44 & -1.44 & -1.43 & -1.42 \\
\hline
\end{tabular}


error when used with MF1. SORM and MCS experienced an improvement that was less pronounced but still significant, with MF1 providing an average MAPE that was 192 and 268 times less for SORM and MCS respectively than the LFM.

In conclusion, the MFMs were found to provide superior accuracy to the LFM for each of the reliability analysis methods investigated with computation times very similar to those of the LFM, or in the case of the indirect models significantly less. The direct MFMs provided reliability indices that were of the order of 50-100 times more accurate than the indirect MFMs and the response surface approximation of the high-fidelity model, $\mathrm{HFM}^{\mathrm{res}}$. The best overall performance was achieved by MF1 which involved coupling the LFM with the ratio response surface $\beta^{\text {res }}(\mathbf{X})$. Results suggest that MF1 could act as a very good substitute for the HFM when used for reliability analysis with MCS, FORM, and SORM.

\section{Conclusions}

In this work, a method for the application of multi-fidelity modeling to the reliability analysis of $2 \mathrm{D}$ elastostatic structures using the BEM is proposed. The reliability of several MFMs created using second-order polynomial response surfaces was evaluated alongside that of a LFM and a HFM using MCS, FORM, and SORM. Results show that, MF1, the MFM that directly coupled the LFM with a ratio correction response surface proved to be the most accurate, providing errors relative to the HFM that were on average 1011, 192, and 268 times smaller than the LFM for FORM, SORM, and MCS, respectively. It was also of similar computational cost, being only $7.95 \%$ slower in the case of MCS. These results suggest that the multifidelity models involving directly calling the LFM could act as good substitutes for the HFM for reliability analysis using MCS, FORM, and SORM.

Future work will seek to apply the proposed methodology to more complex structures, such as anisotropic plates and shells. The IDM will also be investigated.

\section{Acknowledgment}

This research was supported by a grant provided by the Engineering and Physical Sciences Research Council (EPSRC).

\section{References}

1. X. Huang and F. Aliabadi, A boundary element method for structural reliability, Key Eng. Mater. 627 (2015) 453-456.

2. A. Haldar and S. Mahadevan, Probability, Reliability and Statistical Methods in Engineering Design (John Wiley \& Sons, 1999).

3. X. Du, Probabilistic engineering design (2005). Available from http://web.mst.edu/ $\sim$ dux/repository/me360/me360_presentation.html. Accessed on 14 February 2017.

4. C. Su, S. Zhao and H. Ma, Reliability analysis of plane elasticity problems by stochastic spline fictitious boundary element method, Eng. Anal. Bound. Elem. 36(2) (2012) 118-124. 
5. E. D. Leonel and W. S. Venturini, Probabilistic fatigue crack growth using BEM and reliability algorithms, WIT Transactions on Modelling and Simulation 1 (2011) 3-14.

6. X. Huang and M. H. Aliabadi, Probabilistic fracture mechanics by the boundary element method, Int. J. Fracture 171(1) (2011) 51-64.

7. C. Su and J. Xu, Reliability analysis of Reissner plate bending problems by stochastic spline fictitious boundary element method, Eng. Anal. Bound. Elem. 51 (2015) 37-43.

8. M. H. Aliabadi, The Boundary Element Method: Applications in Solids and Structures (John Wiley \& Sons, 2002).

9. L. Yu, P. K. Das and Y. Zheng, A response surface approach to fatigue reliability of ship structures, Ships Offshore Struct. 4(3) (2009) 253-259.

10. S. Hassanien, M. Kainat, S. Adeeb and D. Langer (eds.), On the use of surrogate models in reliability-based analysis of dented pipes, in 11th Int. Pipeline Conf., Calgary, Alberta, Canada (2016).

11. O. S. Lee and D. H. Kim (eds.), Reliability of fatigue damaged structure using FORM, SORM and fatigue model, in World Cong. on Engineering, London, UK (2007).

12. W. Zhao and W. Wang, Application of cokriging technique to structural reliability analysis, in Int. Conf. on Future Computer Sciences and Application (IEEE, 2011), pp. 170-174.

13. G. Sun, G. Li, S. Zhou, W. Xu, X. Yang and Q. Li, Multi-fidelity optimization for sheet metal forming process, Struct. Multidiscip. Optim. 44(1) (2010) 111-124.

14. R. Vitali, R. T. Haftka and B. V. Sankar, Multi-fidelity design of stiffened composite panel with a crack, Struct. Multidiscipl. Optim. 23(5) (2002) 347-356.

15. P. Perdikaris, D. Venturi, J. O. Royset and G. E. Karniadakis, Multi-fidelity modelling via recursive co-kriging and Gaussian-Markov random fields, Proc. Roy. Soc. A Math., Phys. Eng. Sci. 471(2179) (2015) 20150018.

16. A. J. Keane, A. Sóbester and A. I. J. Forrester, Multi-fidelity optimization via surrogate modelling, Proc. Roy. Soc. A: Math., Phys. Eng. Sci. 463(2088) (2007) 3251-3269.

17. B. Glaz, T. Goel, L. Liu, P. Friedmann and R. Haftka, Application of a weighted average surrogate approach to helicopter rotor blade vibration reduction, in 48th AIAA/ASME/ $A S C E / A H S / A S C$ Structures, Structural Dynamics, and Materials Conference (2007), pp. 2370-2394.

18. T. Simpson, V. Toropov, V. Balabanov and F. Viana, Design and analysis of computer experiments in multidisciplinary design optimization: A review of how far we have come or not, in 12th AIAA/ISSMO Multidisciplinary Analysis and Optimization Conf., 10-12 September, Victoria, British Columbia, Canada (2008).

19. A. I. Khuri and S. Mukhopadhyay, Response surface methodology, Comput. Statist. 2(2) (2010) 128-149.

20. R. Rakwitz and B. Fiessler, Structural reliability under combined random load sequences, Comput. Struct. 9(5) (1978) 484-494.

21. K. Breitung, Asymptotic approximations for multinormal integrals, J. Eng. Mech. 110(3) (1984) 357-366.

22. R. Rackwitz, Reliability analysis - a review and some perspectives, Struct. Saf. 23(4) (2001) 365-395.

23. D. Won Kim and B. Man Kwak, Reliability-based shape optimization of two-dimensional elastic problems using BEM, Comput. Struct. 60(1) (1995) 743-750. 